\title{
Politique
}

Politique

\section{Pierre Laplante et Joseph Levy (sous la direction de), La paix : nouvelles avenues, Montréal, Éditions du Méridien, 1987, 186 p.}

\section{France Giroux}

Numéro 14, automne 1988

Sport et politique et Le NPD

URI : https://id.erudit.org/iderudit/040610ar

DOI : https://doi.org/10.7202/040610ar

Aller au sommaire du numéro

Éditeur(s)

Société québécoise de science politique

ISSN

0711-608X (imprimé)

1918-6584 (numérique)

Découvrir la revue

Citer ce compte rendu

Giroux, F. (1988). Compte rendu de [Pierre Laplante et Joseph Levy (sous la direction de), La paix : nouvelles avenues, Montréal, Éditions du Méridien, 1987, 186 p.] Politique, (14), 187-190. https://doi.org/10.7202/040610ar d'utilisation que vous pouvez consulter en ligne.

https://apropos.erudit.org/fr/usagers/politique-dutilisation/ 
Pierre Laplante et Joseph Levy (sous la direction de), La paix: nouvelles avenues, Montréal, Éditions du Méridien, 1987, 186 p.

L'ouvrage, constitué des Actes du colloque «Une deuxième chance pour la paix», regroupe avec bonheur les textes de spécialistes (des politologues pour la plupart) et de promoteurs de la paix (liés soit à des organisations non-gouvernementales, soit aux Nations Unies il y a quelques années). La première partie du volume consiste en une analyse des quarante dernières années afin d'en tirer des leçons pour l'avenir. Daniel Holly y analyse l'échec du projet de sécurité collective qui avait été confié à l'Organisation des Nations Unies. Selon Holly, la nature du système mondial serait l'un des facteurs les plus significatifs de l'échec relatif de la sécurité collective par les Nations Unies. En effet, «il est généralement admis que l'expansion planétaire du capitalisme est à l'origine de la structuration des rapports internationaux en système mondial... Ce système, qui comprend trois sous-systèmes, le soussystème économique, le sous-système politico-militaire et le soussystème idéologique ou socio-culturel, crée des situations où les tensions dans les relations internationales sont inévitables». Ainsi l'«articulation forcée à l'économie mondiale des économies dominées produit des situations de fait qui conduisent peu à peu à des remises en question, ici et là, de cet ordre du monde. D'où l'obligation pour les pays dominants d'intervenir dans les affaires des petits pays pour rétablir le statu quo, ou tenter de le faire" (37). Les tensions ainsi créées dégénèrent et deviennent des conflits armés, comme c'est le cas actuellement en Amérique centrale et en Angola.

En fait, du point de vue du maintien de la paix, l'ONU a été marquée par quarante ans d'impuissance causés par diverses carences dont l'une est sans doute l'absence de volonté d'intégrer suffisamment l'Union soviétique à l'économie mondiale. Somme toute, l'échec relatif des Nations Unies dans le domaine de la 
paix internationale serait causé, avant tout, par la domination économique que les pays riches ont instaurée à l'aide de l'Organisation même. "Ce n'est [certes] pas par hasard, comme le mentionne Holly, que les fonctions économico-sociales de l'ONU se sont tellement développées depuis la fin de la Deuxième Guerre mondiale» (40).

Dans un texte intitulé "Conflits récents dans le Tiers Monde et théories des conflits", Bahgat Korany met l'accent sur la nécessité de concevoir des théories originales afin d'analyser pertinemment les conflits du Tiers Monde. L'auteur cite l'exemple de la guerre Iran-Irak dont les coûts en termes humain et financier sont énormes pour les protagonistes et en déduit que lorsque "les massacres massifs et les destructions à grande échelle s'arrêteront" (14), les coûts seront certes dans les milliers de milliards de dollars. Cet exemple illustre bien que contrairement aux analyses nombreuses qui conçoivent le Tiers Monde comme une "périphérie internationale", cet ensemble de pays doit être conçu - au plan conflictuel - comme le "centre» du système mondial. Selon Korany, il y a, en ce sens, intérêt à développer une approche totalisante de la question, car la militarisation du Tiers Monde fait partie de "l'héritage du système impérial et de ses méthodes d'organisation - ou de désorganisation - du monde». Certains spécialistes n'y voient-ils pas "un élément de la chaîne de dépendance qui se perpétue entre le Nord et le Sud, avec la bénédiction des grandes puissances?» $(27-28)$.

Le texte de Charles-Philippe David a le mérite de répertorier les théories de la dissuasion nucléaire selon quatre catégories fondamentales et d'indiquer la logique qui est propre à chacune. Toutefois, il faudrait prendre en compte à la lecture de ce texte que, depuis son édition, certains accords sur le désarmement modifiant la dissuasion existante, comme celui de décembre 87 lors de la visite du secrétaire général Gorbatchev aux États-Unis, ont été signés. Quoi qu'il en soit, selon David, «la dissuasion 
nucléaire est une réalité avec laquelle on doit inévitablement composer, et ce pour encore très longtemps». Car comme le dit Jonathan Schell, dans son livre The Abolition où il suggère d'abolir purement et simplement les arsenaux nucléaires, «même cette solution exclut à ses yeux l'idée qu'on puisse "oublier» la manière avec laquelle on peut fabriquer des bombes» (54).

Jean-Pierre Derriennic, quant à lui, explique pourquoi les sociétés occidentales sont devenues relativement pacifiées. Il est possible que cette relative pacification soit l'effet de facteurs d'évolution à long terme tel le développement considérable des échanges économiques entre ces pays. «L'optimisme des économistes libéraux du XIX ${ }^{\mathrm{e}}$ siècle, qui [à l'instar de Kant] voyaient dans le commerce international un antidote à la guerre n'est peut-être pas... sans fondement» (84). Un second facteur est sans doute «le nouveau régime démographique résultant du développement économique et des progrès de la médecine». En effet, «dans les sociétés où l'espérance de vie est faible ... les attitudes envers la vie et la mort ne sont pas les mêmes que dans la nôtre». La guerre semble plus odieuse lorsqu'elle devient la seule cause de mortalité collective soudaine; «ce que les famines et les épidémies étaient encore partout au début du $\mathrm{XIX}^{\mathrm{e}}$ siècle» (85). Selon Derriennic, ces mêmes facteurs pourraient contribuer à pacifier les autres sociétés.

La deuxième partie du volume traite de la possibilité de relancer la paix dans la perspective d'une "promotion positive" de la paix plutôt que dans la perspective de la dissuasion armée qui prévaut actuellement. David Mandel présente «une thèse un peu révisionniste, à savoir que les revirements de la politique occidentale envers l'U.R.S.S. ne sont souvent que minimalement liés à des changements réels dans le comportement soviétique" (121). Ainsi, l'escalade des armements soviétiques ne peut être considérée comme étant à la source de l'intensification de l'hostilité Est-Ouest. Car l'Institut international de recherche sur la paix de Stockholm a trouvé que le taux de croissance des dépenses 
militaires soviétiques est resté stable, grosso modo, au cours des années 70 . En outre, rien ne montre qu'il y ait eu une dégradation dans le domaine des droits de la personne depuis la fin des années 60. Les variations de la perception par les États-Unis de la soidisant menace soviétique proviendrait des humeurs de Washington, adaptées à la sauvegarde de la cohésion politique. Le gouvernement américain, de ce point de vue, serait hégélien.

Le constat de la précarité de la détente conduit l'auteur à conclure qu'il ne faut pas compter sur les gouvernements pour mettre fin à la «course aux armements» (127). Il faudrait, en revanche, que les populations exigent une démocratisation de la politique extérieure, à l'Ouest comme à l'Est. Or, «dans ce qu'on est convenu d'appeler les démocraties occidentales, la politique extérieure est la sphère la moins soumise au contrôle démocratique... c'est... la sphère par excellence du secret étatique et de la manipulation» (127-128).

La troisième partie de l'ouvrage porte sur les moyens d'action envisageables pour promouvoir la paix dans les années à venir. Gérard Pelletier abonde dans le sens de Daniel Holly quand il affirme qu'on ne peut ignorer «le lien qui existe, en dépit des apparences, entre la paix et le sous-développement». Pelletier est de ceux qui croient «qu'aussi longtemps qu'on n'aura pas sérieusement entamé le problème du sous-développement dans le monde, on aura très peu de chances de régler les problèmes du désarmement nucléaire, et ensuite du désarmement conventionnel" (165).

L'ouvrage, dans son ensemble, se lit aisément. Son traitement des diverses dimensions de la paix (le rôle de l'opinion publique pour assurer le respect des droits et libertés, la faisabilité de la détente Est-Ouest, la nécessité de la solidarité Nord-Sud) est d'autant plus intéressant que la rédaction de l'ouvrage ne comporte ni longueur ni redondance.

France Giroux

Université de Montréal 